\title{
ANÁLISIS Y ESTUDIO DEL DESEMPEÑO DEL CÓDIGO CHEQUEO DE PARIDAD DE BAJA DENSIDAD IRREGULAR EN UN CANAL DE LÍNEA ELÉCTRICA DE BAJA TENSIÓN
}

\section{ANALYSIS AND STUDY OF THE PERFORMANCE OF THE IRREGULAR LOW DENSITY PARITY CHECK CODE IN LOW VOLTAGE POWERLINE}

\author{
Washington Fernández R. ${ }^{1} \quad$ Cristian Garrido M. $^{2} \quad$ Héctor Olivares L. $^{3}$ \\ Recibido el 10 de enero de 2007, aceptado el 11 de marzo de 2009 \\ Received: January 10, 2007 Accepted: March 11, 2009
}

\begin{abstract}
RESUMEN
En este trabajo se analiza y estudia el código chequeo de paridad de baja densidad irregular (LDPC), en un canal de línea eléctrica de baja tensión. Se analizan los modelos de ruido existentes para las líneas eléctricas de baja tensión.

Se evalúa el desempeño del código LDPC irregular, en un canal de línea eléctrica de baja tensión para diferentes velocidades de transmisión (3, 10, 15 y 30 Mbps), considerando como parámetro de desempeño el BER versus SNR.
\end{abstract}

Palabras clave: Código de chequeo de paridad de baja densidad, ruido en línea eléctrica de baja tensión.

\begin{abstract}
In this work the irregular Low Density Parity Check code (LDPC) is analyzed and studied, is used in a low voltage powerline. Noise models are analyzed for these low voltage lines.

The performance of the LDPC code is evaluated in a low voltage line channel for rates of 3, 5, 15 and 30 Mbps, considering of BER vs SNR a parameter.
\end{abstract}

Keywords: Irregular Low Density Parity Check code, noise in low voltage powerline.

\section{INTRODUCCIÓN}

Las comunicaciones sobre líneas de energía eléctricas se remontan a los años 20, cuando el proceso de electrificación acababa de comenzar [1,2]. Es así como las empresas eléctricas usan su infraestructura con el propósito adicional de telemetría y control de supervisión [1, 3], debido a la falta de disponibilidad de redes telefónicas en posiciones remotas [3].

Para aquello, la modulación lineal y modulación de frecuencia se usaron como las técnicas de modulación primarias [4], en lo que se denomina transmisión de portadora sobre líneas de poder (CTP), con la característica de utilizar frecuencias en el rango de 15 a $500 \mathrm{KHz}$ y bajas potencias de transmisión (hasta $10 \mathrm{Watt}$ ) sobre líneas de alta tensión, cubriendo distancias de entre 160 y $900 \mathrm{~km}$.
A contar de 1930, se usa señalización de portadora (RCS), para comunicar datos telemétricos, esta vez en forma unidireccional, sobre líneas de energía de media y baja tensión [5], empleando técnicas como conmutación de amplitud (ASK) y conmutación de frecuencia (FSK). Los sistemas RCS funcionan con frecuencias de portadora muy cercana a la frecuencia de señal de poder $(100-900 \mathrm{~Hz})$ y tienen velocidades de transferencia de datos sumamente bajas (del orden de unos pocos bits por segundos) a fin de mantener comunicaciones confiables en presencia de la fuerte interferencia de la señal de poder. La ventaja principal de RCS se debe al hecho de que la señal de datos puede pasar fácilmente por los transformadores, sufriendo muy pequeña atenuación y por esto son principalmente usados sobre redes que incorporan un gran número de transformadores inherentes a la red [6].

Nuevos sistemas con bajas tasas de transmisión se desarrollan hasta la década del 80, para aplicaciones de

\footnotetext{
1 Departamento Ingeniería Eléctrica y Electrónica. Universidad del Bío-Bío. Avenida Collao 1202. Concepción, Chile. E-mail: wfernand@ubiobio.cl 2 Departamento Ingeniería Eléctrica y Electrónica. Universidad del Bío-Bío. Avenida Collao 1202. Concepción, Chile. E-mail: cgarrido@ubiobio.cl

3 Departamento Ingeniería Eléctrica y Electrónica. Universidad del Bío-Bío. Avenida Collao 1202. Concepción, Chile. E-mail: holivares@ubiobio.cl
} 
lectura de medidores de energía y de gestión de demanda eléctrica [7]. Las primeras investigaciones con el fin de analizar las características de la red eléctrica y la capacidad de la misma como canal de comunicación se realizan por algunas empresas de energía en Europa y Estados Unidos. Las bandas entre 5 y $500 \mathrm{kHz}$ son las más consideradas y dos factores tienen predominancia en estos estudios: la relación señal a ruido y la atenuación de la señal en la red. Sistemas capaces de establecer comunicaciones en forma bidireccional a través de la red de distribución se obtienen apenas en la década del 90.

Estudios recientes sugieren el uso de frecuencias en el intervalo entre 1 y $30 \mathrm{MHz}$ [8-11].

Los códigos de chequeo de paridad de baja densidad fueron inventados por Gallager en 1963 [12]. Los códigos LDPC son redescubiertos en la mitad del año 1990, con el trabajo de Mackay, Luby, y otros [13-15]. Demostraron que los códigos LDPC son capaces de acercar su desempeño a la capacidad del canal con una baja complejidad de codificación, cuando son decodificados con algoritmos de decodificación iterativa, por ejemplo, el algoritmo suma de producto [16]. Un código LDPC se describe por su matriz de chequeo de paridad $\mathrm{H}$, la cual es una matriz de dimensión m por $n$. El nombre de baja densidad se refiere al hecho que la matriz $\mathrm{H}$ es muy dispersiva, es decir, tiene muchos ceros comparado con la cantidad de unos. Se dice que la longitud del código LDPC es n y su velocidad es $\frac{n-m}{n}$. Dada una matriz de chequeo de paridad $\boldsymbol{H}$, se define un gráfico Tanner [17], asociado $G(H)=(V, E)$, como un gráfico bipartido con $m+n$ vértices, $V=\{1,2,3, \ldots \ldots . m, m+1, \ldots \ldots . m+n\}$, los primeros $m$ vértices corresponde a las $m$ ecuaciones de chequeo de paridad, y son referidas como nodos de chequeo. Los últimos $n$ vértices son referidos como los bits de nodos. Para $l \leq i \leq m$ y $l \leq j \leq n$, hay una unión $(i, m+j)$ en E, sí y solo si $H i, j=1$.

Un código LDPC regular es aquel que tiene todas las columnas con un grado igual, es decir, la misma cantidad de unos por columnas, lo mismo sucede con las filas, en cambio un código LDPC irregular tiene un grupo de columnas con una cierta cantidad de unos y el resto de las columnas con otra cantidad de unos, lo mismo ocurre con las filas.

Como la línea eléctrica de baja tensión es un canal con ruido predominantemente impulsivo, las técnicas de codificación empleadas son las mismas que se emplean para canales con ruido impulsivo (líneas telefónicas conmutadas por ejemplo), estos genéricamente se denominan códigos de números complejos y la modulación OFDM se considera un caso particular de los códigos de números complejos, otros esquemas empleados son la codificación turbo, CDMA.

\section{ANÁLISIS TEÓRICO}

La transmisión de señales de comunicación utilizando las líneas eléctricas como canal de transmisión considera como banda de frecuencias aprovechable al rango que se extiende desde 1,6 MHz hasta los $30 \mathrm{MHz}$ [18], tal como se muestra en la figura 1 .

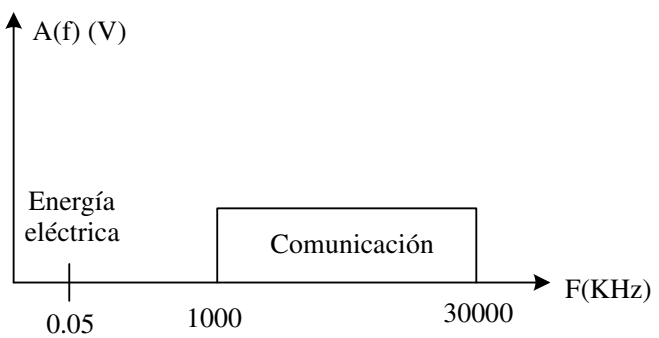

Figura 1. Banda de frecuencias para línea eléctrica de baja tensión.

Los ruidos impulsivos que se encuentran en un canal de línea eléctrica de baja tensión son:

Ruido impulsivo periódico-asíncrono con la frecuencia de la red: En muchos casos se encuentran con una tasa de repetición entre 50 y $200 \mathrm{KHz}$; se generan principalmente por fuentes de poder conmutadas.

Ruido impulsivo periódico-síncrono con la frecuencia de la red: Se presentan con una tasa de repetición de 50 ó 100 $\mathrm{Hz}$ (Europa). Este componente de ruido impulsivo tiene en general una corta duración (10-100 $\mu$ s) y su densidad espectral de potencia (PSD) decrece con la frecuencia. Las fuentes de alimentación, debido a la conmutación de los diodos rectificadores operando en forma síncrona con la red son las principales causantes de este tipo de ruido.

Ruido impulsivo asíncrono: Causado por transientes en la red, presenta duraciones en el rango de microsegundos a unos milisegundos, ocurriendo en forma aleatoria. Debido a estos altos valores, la PSD de este tipo de ruido puede mostrar niveles considerables sobre una PSD de fondo, tal como muestra la figura 2 [19], en la cual se puede observar que en algunas frecuencias un ruido de naturaleza impulsiva supera al ruido de fondo en hasta $50 \mathrm{~dB}$. 


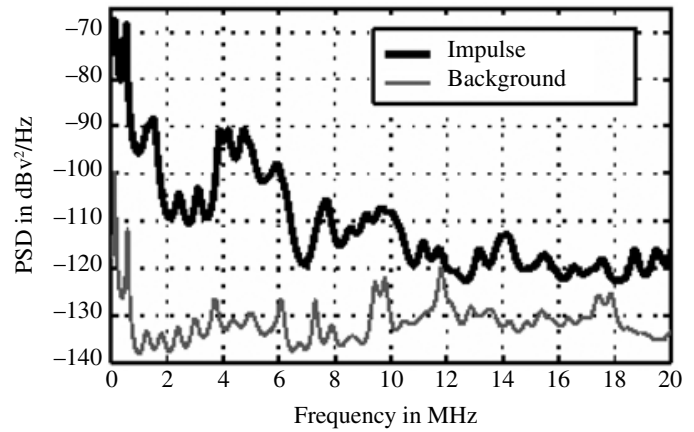

Figura 2. Densidad espectral de potencia de un típico evento con características impulsivas.

A partir de los resultados obtenidos por [19], se presenta un modelo de ruido impulsivo, basado en un tren de impulsos generalizado, de la forma:

$$
n_{i m p}(t)=\sum_{i} A_{i} \operatorname{imp}\left(\frac{t-t_{a_{i}}}{t_{w_{i}}}\right)
$$

Donde:

$n_{\text {imp }}:$ Ruido impulsivo.

$A_{i}$ : Amplitud del impulso i-ésimo.

$\operatorname{imp}\left(\frac{t-t_{a_{i}}}{t_{w_{i}}}\right):$ Tren generalizado de impulsos.

$t_{a_{i}}$ : Tiempo de arribo del impulso i-ésimo.

$t_{w_{i}}:$ Ancho del impulso i-ésimo.

Todos los parámetros de (1) son variables aleatorias que se deben determinar mediante mediciones. Es posible calcular la energía del impulso, de la siguiente manera [19]:

$$
E_{i m p}=\int_{t_{a}}^{t_{a}+t_{w}} n_{i m p}(t)^{2} d t
$$

\section{Donde:}

$E_{\text {imp }}:$ Energía del impulso en Joules.

$n_{\text {imp }}$ : Ruido impulsivo en Volt.

$t_{\mathrm{a}}$ : Tiempo de arribo del impulso en segundo.

$t_{w}$ : Ancho del impulso en segundo.

La potencia de ruido impulsivo se obtiene de [19]:

$$
P_{i m p}=\frac{1}{t_{w}} \int_{t_{a}}^{t_{a}+t_{w}} n_{i m p}(t)^{2} d t
$$

Donde:

$P_{\text {imp }}$ : Potencia de ruido impulsivo en Watt.

Fritchman [20] presenta una forma de caracterizar los canales de línea eléctrica de baja tensión, basados en la utilización de cadenas de Markov, representación de dos estados que se conoce como modelo de Golbert-Elliot. En su estudio, Fritchman propone una representación a través de una cadena de Markov finita, con $n$ estados representando una situación del canal, particionada en un grupo A, con $k$ estados "buenos" (libres de errores) y un segundo grupo B, con $(n-k)$ estados "malos", marcados por la ocurrencia de errores. La figura 3 muestra el modelo de Fritchman.

En [21] se muestra que el ruido no blanco de fondo puede ser modelado como un ruido blanco de banda limitada, y se representa por:

$$
N(f)=10^{\left(k-3.95 \times 10^{-5} f_{o}\right)}
$$

Donde:

$N(f)$ : Densidad espectral de ruido de fondo.

$k$ : Factor de ruido.

$f_{o}$ : Frecuencia de operación.

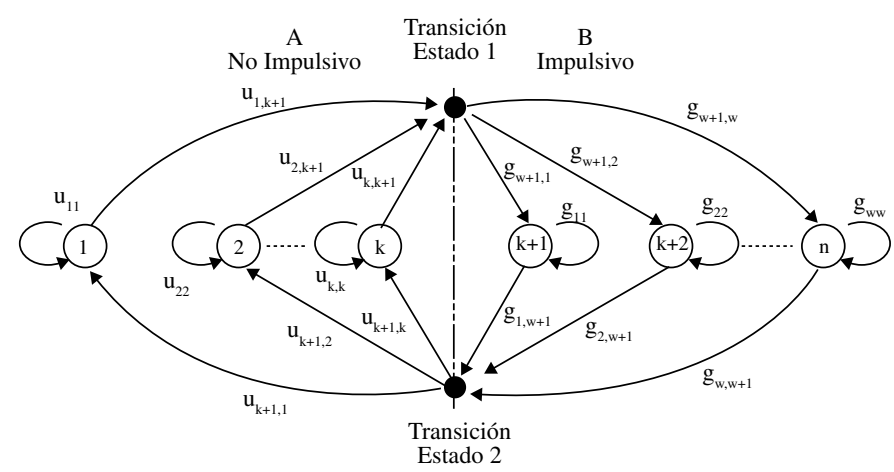

Figura 3. Representación de ruido impulsivo con modelo particionado de Markov, de acuerdo a Fritchman. 
De acuerdo a [21], la densidad espectral de potencia de ruido no blanco de fondo se muestra en la figura 4.

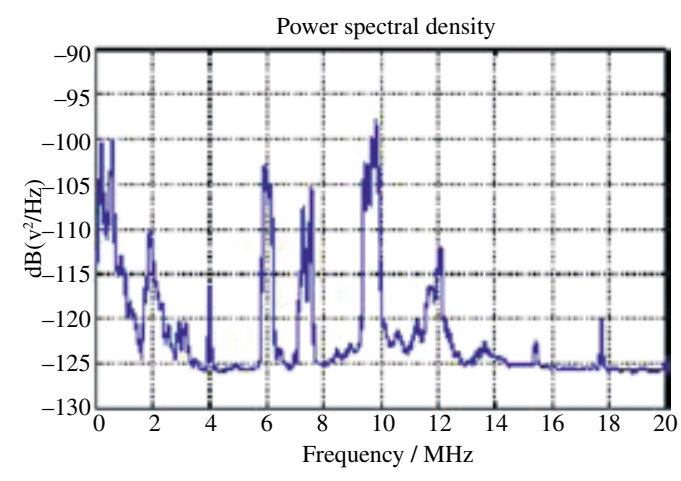

Figura 4. Densidad espectral de ruido no blanco de fondo.

Middleton [22] propone en 1977 un modelo de ruido impulsivo, que está formado por la suma de ruido blanco y ruido impulsivo. En este modelo, según el ancho de banda del ruido, éste se clasifica en tres clases generales: A, B y C.

Ruido clase A: Interferencia cuyos espectros son iguales o más estrechos que el ancho de banda del receptor.

Ruido clase B: Ruido cuyos espectros son más amplios que el ancho de banda del receptor.

Ruido clase C: Interferencia que incluye las características de clase A y B.

De acuerdo a [22], el ruido presente en las líneas eléctricas es del tipo clase A.

La varianza $\sigma^{2}$ del ruido se representa por la suma de las varianzas de la componente Gaussiana y la componente impulsiva [23]. Es decir:

Donde:

$$
\sigma^{2}=\sigma_{G}^{2}+\sigma_{i}^{2}
$$

$\sigma^{2}$ : Varianza del ruido de línea eléctrica de baja tensión. $\sigma_{G}^{2}$ : Varianza de la componente Gaussiana del ruido.

$\sigma_{i}^{2}$ : Varianza de la componente impulsiva del ruido.

La función de densidad de probabilidad (FDP), del ruido de Middleton clase A, se representa por [23]:

$$
p_{z}(z)=\sum_{m=0}^{\infty} \frac{e^{-A} A^{m}}{m !} \frac{1}{\sqrt{2 \pi \sigma_{m}^{2}}} e^{\frac{-|z|^{2}}{2 \sigma_{m}^{2}}}
$$

Donde:

z: Envolvente del ruido.

$\sigma_{m}^{2}$ : Varianza de Middleton.

$m$ : Número de subcanales de Middleton.

$A$ : Índice impulsivo.

El parámetro $A$ se define por el producto entre la duración promedio de los impulsos y la razón de generación media del ruido impulsivo (cantidad de eventos impulsivos por unidad de tiempo).

La varianza de Middleton se obtiene a partir de [23]:

$$
\sigma_{m}^{2}=\sigma^{2} \frac{\frac{m}{A+\Gamma}}{1+\Gamma}
$$

Donde:

$\Gamma$ : Relación entre las potencias Gaussiana e impulsiva, y está dada por [23]:

$$
\Gamma=\frac{\sigma_{G}^{2}}{\sigma_{i}^{2}}
$$

Esto significa que el modelo de Middleton se representa por el producto de una distribución Poisson, $P(m ; A) \mathrm{y}$ una distribución de Gauss, $G\left(z, \sigma_{m}^{2}\right)$. Esto es:

$$
p_{z}(z)=\sum_{m=0}^{\infty} P(m ; A) G\left(z ; \sigma_{m}^{2}\right)
$$

Donde:

$$
P(m ; A)=\frac{e^{-A} A^{m}}{m !}
$$

$\mathrm{y}$

$$
G\left(z ; \sigma_{m}^{2}\right)=\frac{1}{\sqrt{2 \pi \sigma_{m}^{2}}} e^{-\frac{\left|z^{2}\right|}{2 \sigma_{m}^{2}}}
$$


El modelo de Middleton clase A consiste de la sumatoria de infinitos ruidos Gaussianos, cada uno con distinta potencia de ruido. Aquellos que presentan mayor potencia son denominados impulsivos y los que tienen una potencia de ruido mucho menor a los denominados impulsivos (al menos 10 veces, según [23]) se definen como Gaussianos. La distribución de Poisson representa la probabilidad de pasar de un ruido más impulsivo a uno menos impulsivo y viceversa.

De acuerdo a [23], la potencia de ruido Gaussiano y su varianza se relacionan por:

$$
N_{G o}=2 \sigma_{G}^{2}
$$

Como el modelo de Middleton se compone de ruido Gaussiano y ruido impulsivo, ambos con una distribución de Gauss, se cumple que:

$$
N_{o}=N_{G o}+N_{i o}=2 \sigma_{G}^{2}+2 \sigma_{i}^{2}=2 \sigma
$$

Donde:

$N_{o}$ : Densidad de potencia de ruido de Middleton.

$N_{G o}$ : Densidad de potencia de ruido Gaussiano.

$N_{i o}$ : Densidad de potencia de ruido impulsivo.

El algoritmo que se utiliza para la simulación del ruido de línea eléctrica de baja tensión, incluye dos subcanales equiprobables de Middleton. La variable $\sigma_{G}^{2}$ representa la varianza del ruido Gaussiano de fondo y $\sigma_{i}^{2}$ (10 veces mayor a $\sigma_{G}^{2}$ ), la varianza del subcanal impulsivo. Como ambos subcanales presentan una distribución de Gauss, se emplea el algoritmo de box-Muller [24], para modelar el ruido presente en las líneas eléctricas de baja tensión.

La matriz de chequeo de paridad del código LDPC $(\boldsymbol{H})$, el número de uniones que inciden en $v_{i}$, se denomina el grado de la variable nodo $d\left(v_{i}\right)$, es igual al número de unos en la columna $i$, de la matriz de chequeo de paridad. Similarmente el número de uniones que se conectan con $c_{j}$, se denomina el grado del nodo de chequeo $d\left(c_{j}\right)$ y es igual al número de unos en la fila $\mathrm{j}$. Los $d_{v_{\max }} \mathrm{y} d_{c_{\max }}$ corresponden a los grados máximos de variable de nodo y de los nodos de chequeo respectivamente. Sea $\lambda_{i}$ y $\rho_{i}$, que representan la fracción de uniones, que salen desde la variable nodo y nodo de chequeo de grado $i$. Se define:

$$
\lambda(x)=\sum_{i=2}^{d_{v_{\max }}} \lambda_{i} x^{i-1}
$$

Y

$$
\rho(x)=\sum_{i=2}^{d_{c_{\max }}} \rho_{i} x^{i-1}
$$

Son el grado de distribución de la variable nodo y el grado de distribución de nodo de chequeo, respectivamente.

Un ciclo en un gráfico de Tanner está definido como: una ruta desde una variable nodo $v_{i}$, y retorna al mismo lugar, si cualquier unión en la ruta se usa sólo una vez. La longitud de un ciclo es el número de uniones que contiene el ciclo.

La longitud más pequeña de un ciclo en un gráfico se denomina "ciclos mínimos". La importancia de grandes ciclos [25], en los códigos LDPC irregulares cuando se emplea decodificación suma de producto, es que el número de iteraciones independientes del algoritmo es proporcional al ciclo mínimo del gráfico bipartido correspondiente al código. A continuación se da un ejemplo de una matriz de chequeo de paridad con tres unos por columnas y cuatro unos por filas.

$$
\boldsymbol{H}=\left[\begin{array}{l}
001001110000 \\
110010000001 \\
000100001110 \\
010001100100 \\
101000010010 \\
000110001001 \\
100110100000 \\
000001010011 \\
011000001100
\end{array}\right]
$$

La matriz de chequeo de paridad $\boldsymbol{H}$ satisface la siguiente ecuación:

$$
\boldsymbol{H} \boldsymbol{s}^{T}=0
$$

Donde:

$\boldsymbol{H}$ : Matriz chequeo de paridad.

$s$ : Palabra de código a transmitir.

La figura 5 muestra el gráfico bipartido correspondiente a la matriz chequeo de paridad, dado en el ejemplo.

La figura 6 muestra un ciclo de longitud 6 , la ruta que se sigue es: $x_{1}, q_{1}, x_{3}, q_{3}, x_{5}, q_{6}, x_{1}$. 


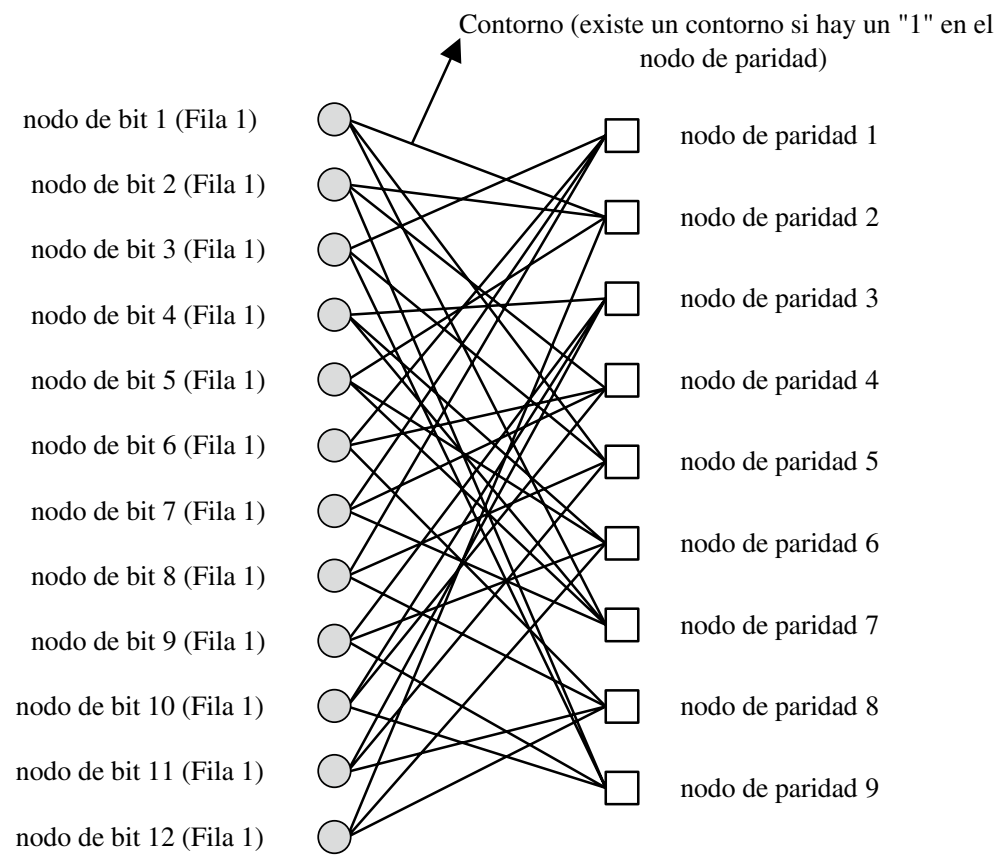

Figura 5. Gráfico bipartido correspondiente a la matriz de chequeo de paridad, del ejemplo.

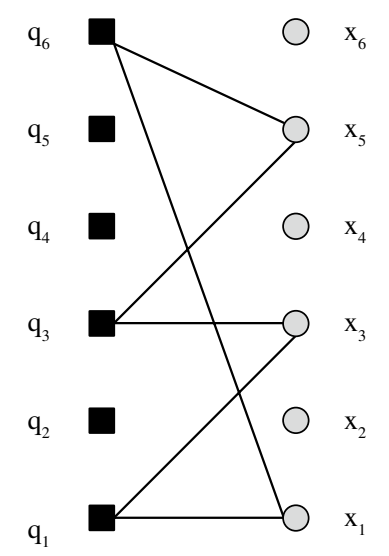

Figura 6. Un ciclo de longitud 6.

Para la decodificación de los códigos irregulares existen varios algoritmos, entre los cuales se pueden mencionar: suma de producto, "belief" propagación, etcétera. A continuación se describirán los pasos que sigue el decodificador suma de producto [24], mediante un ejemplo: supóngase que se tiene una matriz generadora $\boldsymbol{G}$, de la forma:

$$
\boldsymbol{G}=\left[\begin{array}{l}
100001 \\
010100 \\
001010
\end{array}\right]
$$

y una palabra de mensaje $s=[100]$ a transmitir.
Al multiplicar $\boldsymbol{s}$ por $\boldsymbol{G}$ se tiene la palabra código: $\boldsymbol{y}=[1001]$. La multiplicación se realiza en aritmética módulo 2. Esta palabra se envía a través del canal de transmisión, el cual le suma ruido a la señal transmitida; en el receptor se recibe como $\boldsymbol{r}=\boldsymbol{y}+\boldsymbol{n}$, donde $\boldsymbol{n}$, es el vector de ruido del canal de línea eléctrica de baja tensión. El receptor toma la palabra recibida $\boldsymbol{r}$, y debe estimar la palabra transmitida $\boldsymbol{y}$. La decodificación LDPC tiene tres partes principales: la probabilidad inicial, el chequeo de síndrome y la decodificación iterativa. Lo primero que se realiza es convertir la palabra de código $s$ en probabilidad de símbolo. El próximo paso es el chequeo de síndrome, para determinar si $s$ es una palabra de código válida. $\mathrm{Si}$ $\boldsymbol{s}$ es una palabra válida, entonces el mensaje se saca del 
decodificador y la decodificación se terminó. Si no es así, se debe enviar al decodificador iterativo, el cual actualiza las probabilidades, haciendo uso de otras probabilidades. Después de cada iteración se vuelve hacer uso del chequeo de síndrome, para ver si la palabra válida ha sido decodificada. Estas iteraciones se repiten tantas veces como el número de iteraciones ha sido dado, si se cumple este máximo y si el síndrome da un valor distinto de cero, la palabra tiene error que no es posible corregir.

Cuando se usa transmisión binaria, con 1 y 0 , las probabilidades son: $q^{1}$, para indicar que la probabilidad del bit 1 ha sido evaluada y $q^{0}$, la probabilidad del bit 0 ha sido evaluada. Una vez que todos $\operatorname{los} q^{1}$ y $q^{0}$ son evaluados, los chequeos de síndromes y la decodificación iterativa comienza. El chequeo de síndrome toma la probabilidad evaluada, y decide por el 1 ó 0 , de acuerdo a la más alta probabilidad. Utiliza la matriz de chequeo de paridad $\boldsymbol{H}$ para decidir si es una palabra de código válida. Del ejemplo anterior se tiene que la matriz $\boldsymbol{H}$ tiene la forma:

$$
\boldsymbol{H}=\left[\begin{array}{l}
010100 \\
001010 \\
100001
\end{array}\right]
$$

Si la palabra se recibe sin error se tiene que $\boldsymbol{y} \boldsymbol{H}^{T}=[000]$, por lo tanto, se detiene el proceso porque corresponde a la palabra código. Si la palabra llega con error, por ejemplo: sea $m=$ [100001]. Si se multiplica $\boldsymbol{m} \boldsymbol{H}^{T}=$ [001]. Como no es una palabra válida, se debe utilizar el proceso de decodificación iterativa.

El proceso de decodificación iterativa toma las probabilidades desde la probabilidad inicial. Se forma desde la matriz $\boldsymbol{H}$, el gráfico de paso de mensaje, para el ejemplo dado, se da en la figura 7.

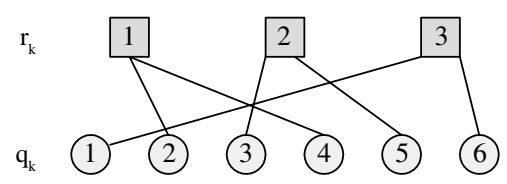

Figura 7. Paso de mensaje para el ejemplo dado.

Mirando la matriz $\boldsymbol{H}$ se observa que en la primera fila tiene 1 en las posiciones segunda y cuarta; existe una conexión entre el primer cuadrado y el segundo y cuarto círculo de la figura 7; las conexiones adicionales son hechas de la misma forma. La idea en la decodificación es iterativamente, volver hacia atrás y adelante, entre las probabilidades $q$ y $r$, para de esta forma obtener en lo posible el mejor resultado. Los $q$ `s, son las probabilidades iniciales, los $r$ `s, son calculados utilizando [24]:

$$
\begin{aligned}
& r_{k}^{0}=\left[1+\prod\left(q_{k}^{0}-q_{k}^{1}\right)\right] \\
& r_{k}^{1}=\left[1-\prod\left(q_{k}^{0}-q_{k}^{1}\right)\right]
\end{aligned}
$$

El $q_{k}^{l}$ indica la probabilidad que el círculo (indicado en la figura 7) $k$ es $1, \mathrm{y} q_{k}^{0}$ indica que la probabilidad en el circulo $k$ es 0 . Las diferencias de los $q$ 's son multiplicadas para obtener los $r$ correspondientes, del ejemplo dado; para determinar $r_{1}^{1}$ se debe multiplicar por $\left(q_{2}^{0}-q_{2}^{1}\right)\left(q_{4}^{0}-q_{4}^{1}\right)$ y restarle 1 . Una vez que todos los $r$ han sido calculados, es posible actualizar los $q$, utilizando [24]:

$$
\begin{gathered}
q_{k}^{0}=\alpha_{k} p_{j}^{0} \prod r_{k}^{0} \\
q_{k}^{l}=\alpha_{k} p_{j}^{l} \prod r_{k}^{l}
\end{gathered}
$$

Donde:

$\alpha_{k}$ : Constante para asegurar que las probabilidades de $q_{k}^{0}$ más $q_{k}^{l}$ sumen 1 .

Con estas cuatro ecuaciones es posible en forma iterativa volver hacia atrás y adelante, para obtener el mejor valor de $q$. Una vez que se recuperan todos los mensajes, la decodificación finaliza.

Para comprender el algoritmo de decodificación LDPC se dará un ejemplo práctico, cuyos resultados se obtuvieron del decodificador implementado. Los resultados fueron redondeados a tres cifras significativas: Suponga que se envía desde el transmisor una secuencia de bits codificado, de la siguiente forma: $[1,1,0,0,1,0,1]$, producto del canal, a los bits se le suma ruido del canal de línea eléctrica de baja tensión, por lo tanto, en el receptor se recibe la señal como: $[-0,56,0,97,-1,20,-3,09,1,34,-2,37$, 2,84 , considere como densidad espectral de ruido un valor de $\sigma^{2}=1.2$.

La matriz de chequeo de paridad $\boldsymbol{H}$ tiene la forma:

$$
\boldsymbol{H}=\left[\begin{array}{lllllll}
1 & 0 & 0 & 1 & 0 & 1 & 1 \\
0 & 1 & 0 & 1 & 1 & 1 & 0 \\
0 & 0 & 1 & 0 & 1 & 1 & 1
\end{array}\right]
$$


La razón de probabilidad para cada $r_{j}$ recibido de acuerdo a:

$$
\Delta_{j}=\frac{p\left(\boldsymbol{r}_{j} \mid \boldsymbol{x}_{j}=+1\right)}{p\left(\boldsymbol{r}_{j} \mid \boldsymbol{x}_{j}=-1\right)}=e^{-\frac{\left(r_{j}\right)}{\sigma^{2}}}
$$

es:

$\Delta_{j}=[0,39,5,07,0,14,0,01,9,25,0,02,114]$

Se crea la matriz $\Delta_{i j}$, asignando los valores de $\Delta_{\mathrm{j}}$, en las columnas $j$, de la matriz de chequeo de paridad, que tenga valores distintos de 0 .

$\Delta_{i j}=\left[\begin{array}{ccccccr}0,39 & 0 & 0 & 0,01 & 0 & 0,02 & 114 \\ 0 & 5,07 & 0 & 0,01 & 9,25 & 0,02 & 0 \\ 0 & 0 & 0,14 & 0 & 9,25 & 0,02 & 114\end{array}\right](27)$

Se realiza el proceso iterativo:

En el paso horizontal se aplica:

$$
\Lambda_{i j}=\prod_{j^{\prime} \varepsilon N(i) / j} \frac{1-\Delta_{i j^{\prime}}}{1+\Delta_{i j^{\prime}}}
$$

Para calcular $\Lambda_{i j}$ :

$$
\left[\begin{array}{ccccccc}
-0,94 & 0 & 0 & -0,41 & 0 & -0,43 & 0,42 \\
0 & -0,77 & 0 & 0,52 & 0,64 & 0,53 & 0 \\
0 & 0 & 0,76 & 0 & -0,72 & 0,60 & -0,59
\end{array}\right]
$$

En el paso vertical se aplica:

$$
\Delta_{i j}=\frac{p\left(\boldsymbol{r}_{j} \mid \boldsymbol{x}_{j}=+1\right)}{p\left(\boldsymbol{r}_{j} \mid \boldsymbol{x}_{j}=+1\right)} \prod_{i^{\prime} \varepsilon M(j) / i} \frac{1-\Lambda_{i^{\prime} j}}{1+\Lambda_{i^{\prime} j}}
$$

Para calcular $\Delta_{i j}$

$$
\left[\begin{array}{lllllc}
0,39 & 0 & 0 & 0,00 & 0 & 442 \\
0 & 5,07 & 0 & 0,02 & 57,1 & 0 \\
0 & 0 & 0,14 & 0 & 41,8 & 46,8
\end{array}\right]
$$

Para calcular la seudo razón de probabilidad, se aplica:

$$
\Delta_{j}=\frac{p\left(\boldsymbol{r}_{j} \mid \boldsymbol{x}_{j}=+1\right)}{p\left(\boldsymbol{r}_{j} \mid \boldsymbol{x}_{j}=+1\right)} \prod_{i \varepsilon M(j)} \frac{1-\Lambda_{i j}}{1+\Lambda_{i j}}
$$

Se tiene:

$$
[11,6,38,2,0,02,0,01,259,0,00,182]
$$

Utilizando las condiciones: $\Delta j \leq 1$ se asigna el bit $0 \mathrm{y}$ $\Delta j>1$ se asigna el bit 1 , el $\boldsymbol{x}$ estimado es $[1,1,0,0,1,0,1]$. Aplicando el síndrome de chequeo $x H^{\mathrm{T}}$, el resultado es 0 , lo que quiere decir que los bits estimados están correctos, y la decodificación finalizó. Si el resultado de síndrome hubiera sido distinto de 0 , se determina $\Delta_{i j}$ y se calcula $\Delta_{j}$, para el nuevo proceso iterativo, las nuevas razones de probabilidades, son las $\Delta_{i j}$ siguientes:

$$
\left[\begin{array}{lllllll}
11,6 & 0 & 0 & 0,01 & 0 & 0 & 182 \\
0 & 38,2 & 0 & 0,01 & 259 & 0,00 & 0 \\
0 & 0 & 0,02 & 0 & 259 & 0,00 & 182
\end{array}\right](34)
$$

Se va al paso horizontal, y al final de nuevo se aplica el chequeo de síndrome; si el resultado es distinto de 0 se repite el proceso, un número dado de repeticiones, que puede ser por ejemplo 100 veces, si se cumple este número y el síndrome da como resultado distinto de 0 , quiere decir que la decodificación falló.

El algoritmo utilizado para construir el código LDPC irregular es el siguiente: primero se selecciona un "perfill", que describe el número requerido de peso para cada columna y el número requerido de peso por fila. La matriz de chequeo de paridad se define con un gráfico bipartido con vértices de nodos que corresponden a las columnas y vértices de chequeo que corresponden a las filas. Segundo, se escoge un "método de construcción", en este caso se utiliza el método de permutaciones, es decir, superponiendo matrices permutadas aleatoriamente [12]. Para efectuar estas permutaciones se utiliza la distribución de Poissons con la característica que la distribución con más altos pesos de las columnas tiene la menor varianza en la distribución de Poisson. En la matriz de chequeo de paridad que se utiliza en este trabajo, las columnas tienen pesos de tres y cuatro y las filas tienen un peso de siete.

La figura 8 muestra el esquema para la simulación del sistema de transmisión y recepción con codificador de línea en un canal de línea eléctrica de baja tensión, el cual es el sistema de referencia para compararlo con el desempeño del sistema código LDPC irregular. 


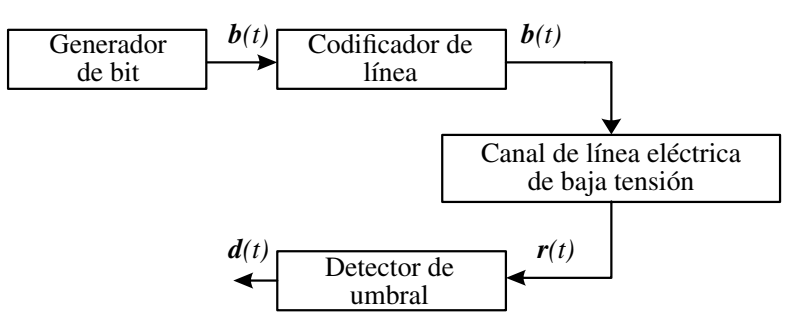

Figura 8. Diagrama en bloques del sistema de transmisión y recepción con codificador de línea en canal de línea eléctrica de baja tensión.

De acuerdo a la figura 8, el bloque generador de bits $\boldsymbol{b}(t)$ representa los bits de datos a transmitir, los cuales pasan por un codificador de línea, que convierte el dato digital en una señal bipolar $\boldsymbol{y}(t)$, asignando un valor "- 1 " a los " 0 's" y un valor " $+l$ " a los " $l$ 's". Esta señal es la que se transmite a través del canal de línea eléctrica de baja tensión. La señal recibida $\boldsymbol{y}(t)$ es la señal transmitida más el ruido del canal de línea eléctrica de baja tensión. Se tiene:

$$
r(t)=y(t)+n(t)
$$

Donde:

$\boldsymbol{r}(t)$ : Señal recibida.

$\boldsymbol{y}(t)$ : Señal transmitida.

$\boldsymbol{n}(t)$ : Ruido de la línea eléctrica de baja tensión.

En el receptor se usa un detector de umbral para estimar los datos transmitidos $\boldsymbol{d}(t)$, el criterio de decisión es el siguiente: valores mayores e iguales a cero, corresponde a un " $l$ " y valores menores que cero, corresponde a un " 0 ".

La figura 9 muestra el diagrama en bloque del esquema propuesto para la simulación de la codificación LDPC irregular en un canal de línea eléctrica de baja tensión.

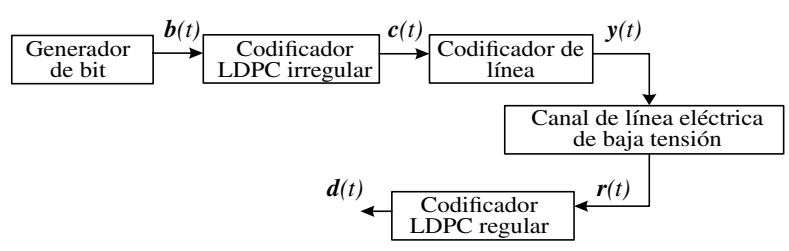

Figura 9. Diagrama en bloque del esquema propuesto para la simulación de la codificación LDPC irregular, en un canal de línea eléctrica de baja tensión.
De acuerdo a la figura 9 , el generador de bits $\boldsymbol{b}(t)$ proporciona los datos a transmitir, el bloque codificador LDPC irregular le suma bits de acuerdo a la matriz generadora de código LDPC irregular $\boldsymbol{c}(t)$. En el codificador de línea a los 0 's le corresponden el valor " -1 " y a los 1 's le corresponden el valor " $+l$ ", $\boldsymbol{y}(t)$. El bloque canal corresponde a la línea eléctrica de baja tensión, que le suma ruido a la señal transmitida $\boldsymbol{r}(t)$. El decodificador LDPC irregular detecta y corrige los errores causados por la presencia de ruido en el canal de transmisión.

\section{RESULTADOS Y DISCUSIÓN DE RESULTADOS}

\section{Resultados}

Las figuras 10 y 11 muestran dos simulaciones de ruido de línea eléctrica de baja tensión modelado en este trabajo. Los parámetros de simulación son $m=2, \sigma_{G}^{2}=1 \mathrm{~mW}$, y $\sigma_{i}^{2}=1 W, A=0.1, \Gamma=-30 \mathrm{~dB}$ y muestras de $200 \mathrm{y}$ 1.000 .

Las figuras 12 y 13 muestran dos simulaciones de ruido de línea eléctrica de baja tensión con $m=1, \sigma_{G}^{2}=1 \mathrm{~mW}$, y $\sigma_{i}^{2}=1 \mathrm{~W}, A=0.15, \Gamma=-30 \mathrm{~dB}$, con muestras de 200 y 1.000 .

Las figuras 14 y 15 muestran simulaciones de ruido de línea eléctrica de baja tensión, con $m=2, \sigma_{G}^{2}=1 \mathrm{~mW}$, y $\sigma_{i}^{2}=5 W, A=0.15, \Gamma=-30 \mathrm{~dB}$, con 200 y 1.000 muestras.

La figura 16 muestra los resultados de la simulación de BER versus SNR, para distintas velocidades de transmisión (3, 10, 15 y 30 Mbps), con codificación de línea.

Las figuras 17, 18, 19 y 20 muestran los resultados de la simulación para las velocidades de transmisión 3, 10, 15 y 30 Mbps, con codificación LDPC irregular, en un canal de línea eléctrica de baja tensión en comparación a la codificación de línea.

La figura 21 muestra el resultado de la comparación para distintas velocidades de transmisión $(3,10,15$ y 30 Mbps), con codificación LDPC irregular en un canal de línea eléctrica de baja tensión. 


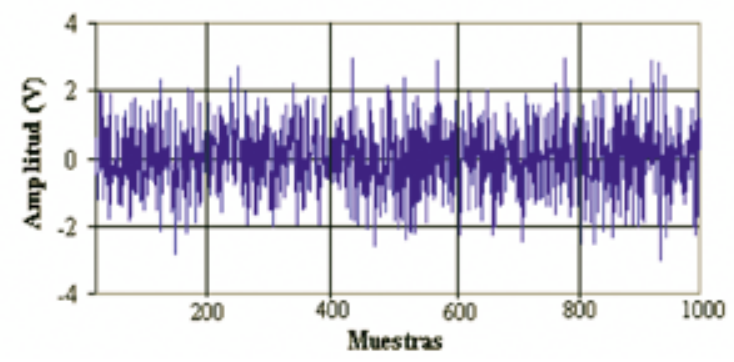

Figura 10. Ruido de línea eléctrica de baja tensión con parámetros $m=2, \sigma_{G}^{2}=1 \mathrm{~mW},{ }_{\mathrm{y}}^{2}=1 \mathrm{~W}$, $A=0,1, \Gamma=-30 \mathrm{~dB}$ y 1.000 muestras.

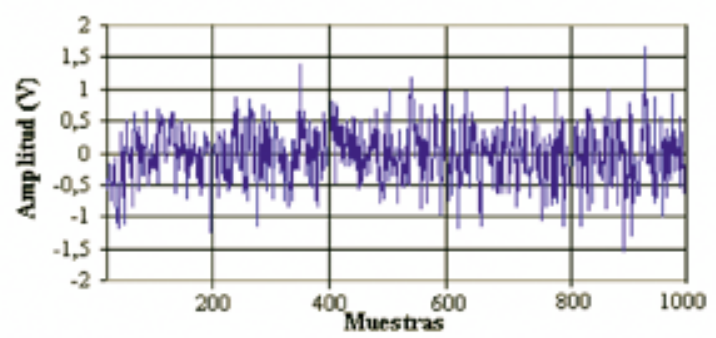

Figura 12. Ruido de línea eléctrica de baja tensión con parámetros $m=1, \sigma_{G}^{2}=1 \mathrm{~mW},{ }_{\mathrm{y}}^{2}=1 \mathrm{~W}$, $A=0,25, \Gamma=-30 \mathrm{~dB}$ y 1.000 muestras.

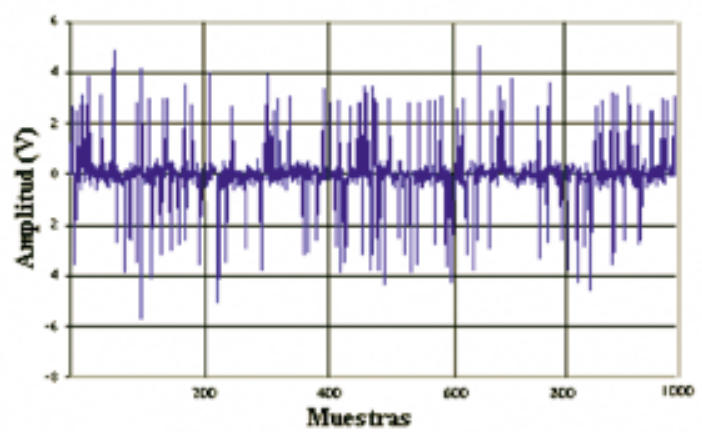

Figura 14. Ruido de línea eléctrica de baja tensión, con $m=2, \sigma_{G}^{2}=1 \mathrm{~mW}$, y $\sigma_{i}^{2}=5 W, A=0,25$, $\Gamma=-30 \mathrm{~dB}$ y 1.000 muestras.

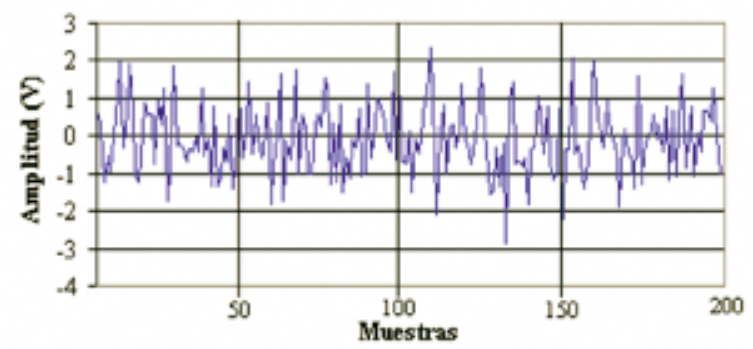

Figura 11. Ruido de línea eléctrica de baja tensión con parámetros $m=2, \sigma_{G}^{2}=1 \mathrm{~mW},{ }_{\mathrm{y}} \sigma_{i}^{2}=1 \mathrm{~W}$, $A=0,1, \Gamma=-30 \mathrm{~dB}$ y 200 muestras.

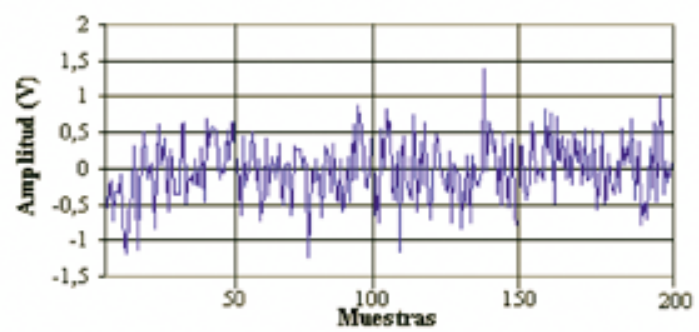

Figura 13. Ruido de línea eléctrica de baja tensión con parámetros $m=1, \sigma_{G}^{2}=1 \mathrm{~mW},{ }_{\mathrm{y}} \sigma_{i}^{2}=1 \mathrm{~W}$, $A=0,1, \Gamma=-30 \mathrm{~dB}$ y 200 muestras.

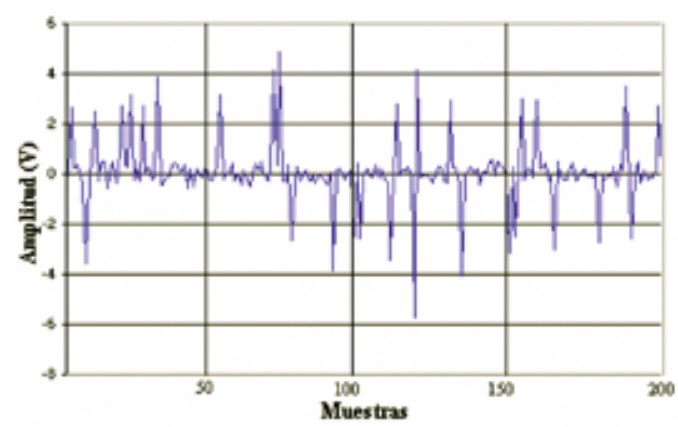

Figura 15. Ruido de línea eléctrica de baja tensión, con $m=2, \sigma_{G}^{2}=1 \mathrm{~mW}$, y $\sigma_{i}^{2}=5 \mathrm{~W}, A=0,25$, $\Gamma=-30 \mathrm{~dB}$ y 200 muestras. 


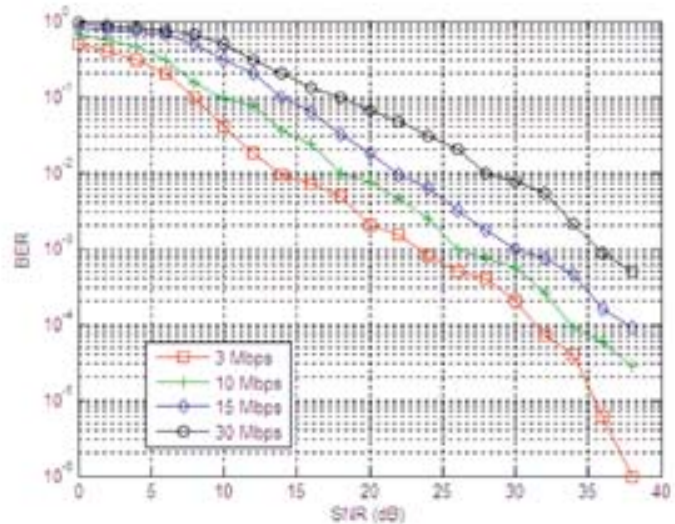

Figura 16. BER versus $\mathrm{SNR}(\mathrm{dB})$, para distintas velocidades de transmisión (3, 10, 15 y $30 \mathrm{Mbps})$, con codificación en línea.

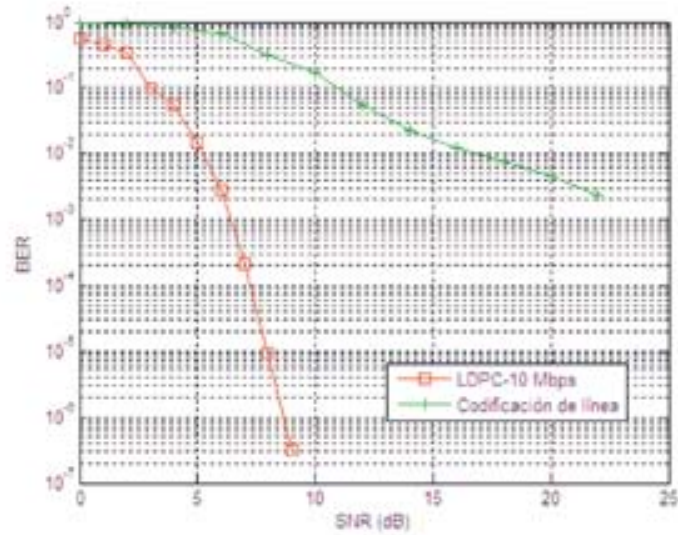

Figura 18. Resultado de la simulación para la velocidad de transmisión $10 \mathrm{Mbps}$, con codificación LDPC irregular, en un canal de línea eléctrica de baja tensión en comparación a la codificación de línea.

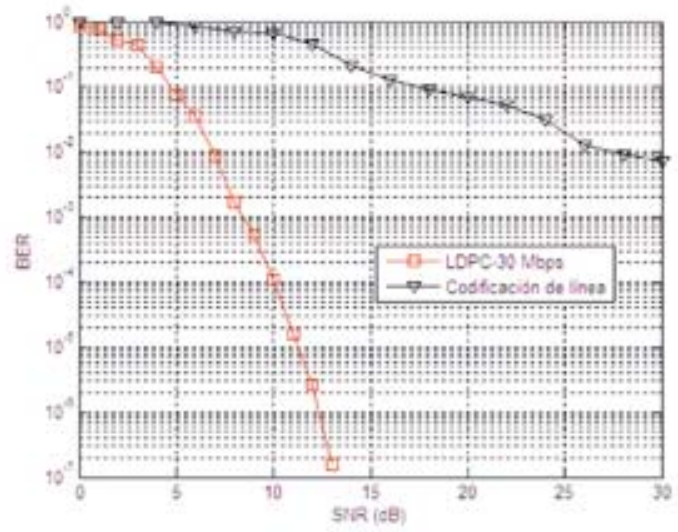

Figura 20. Resultado de la simulación para la velocidad de transmisión 30 Mbps, con codificación LDPC irregular, en un canal de línea eléctrica de baja tensión en comparación a la codificación de línea.

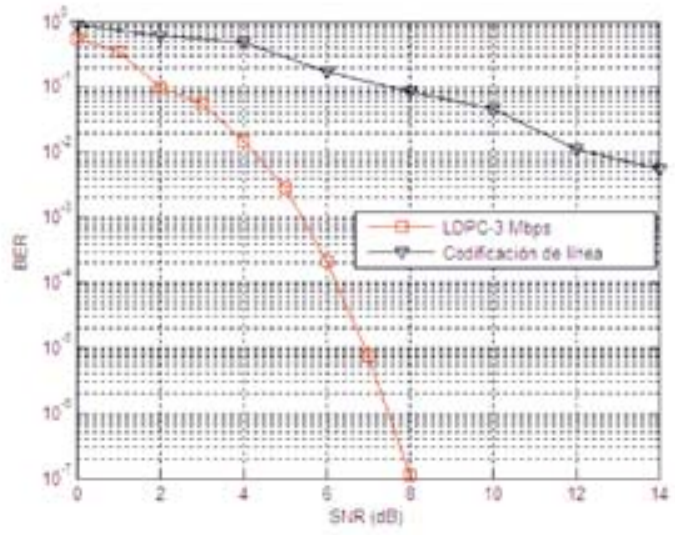

Figura 17. Resultado de la simulación para la velocidad de transmisión 3 Mbps, con codificación LDPC irregular, en un canal de línea eléctrica de baja tensión en comparación a la codificación de línea.

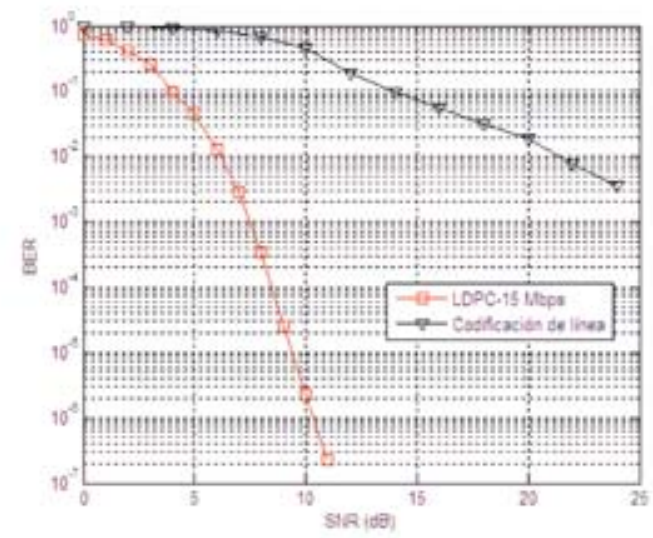

Figura 19. Resultado de la simulación para la velocidad de transmisión 15 Mbps, con codificación LDPC irregular, en un canal de línea eléctrica de baja tensión en comparación a la codificación de línea.

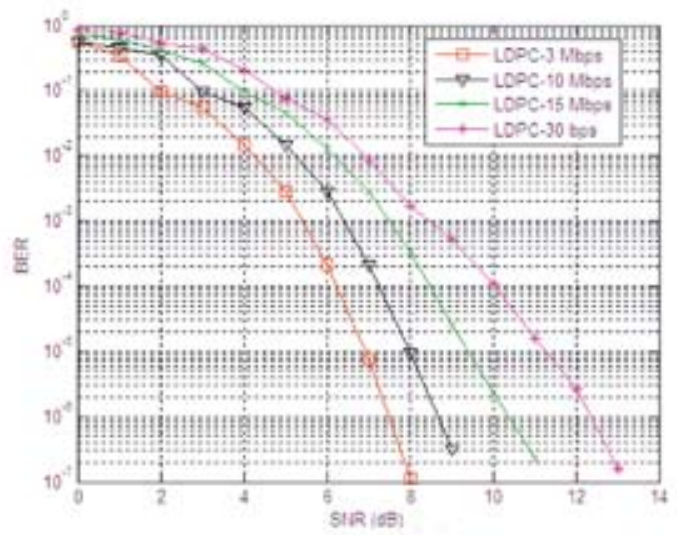

Figura 21. Resultado de la comparación para distintas velocidades de transmisión ( $3,10,15$ y 30 Mbps), con codificación LDPC irregular en un canal de línea eléctrica de baja tensión. 


\section{Discusión de resultados}

De las figuras 10 a 13 se observa que una disminución en el número de fuentes de ruido (parámetro $m$ ) hace menos ruidoso el canal.

De las figuras 10 a 15 se observa que a menor valor de $\Gamma$, el ruido es más impulsivo.

Los resultados obtenidos, que corresponden a los gráficos 16 a 20, se resumen en la tabla 1, la cual da la comparación de la codificación LDPC irregular y codificación de línea para las distintas velocidades de transmisión $(3,10,15$ y $30 \mathrm{Mbps}$ ), considerando un BER de $10^{-6}$.

Tabla 1. Desempeño de la codificación LDPC irregular en comparación a codificación de línea, considerando un BER $10^{-6}$.

\begin{tabular}{|c|c|c|c|}
\hline $\begin{array}{c}\text { Velocidad } \\
\text { Mbps }\end{array}$ & $\begin{array}{c}\text { SNR (dB) } \\
\text { codificación } \\
\text { de línea }\end{array}$ & $\begin{array}{c}\text { SNR (dB) } \\
\text { LDPC } \\
\text { irregular }\end{array}$ & $\begin{array}{c}\text { Ganancia } \\
\text { de código } \\
\text { LDPC } \\
\text { irregular }\end{array}$ \\
\hline 3 & 38 & 6,8 & 31,2 \\
\hline 10 & 43,5 & 9 & 34,5 \\
\hline 15 & 48 & 10,5 & 37,5 \\
\hline 30 & 51,5 & 13,5 & 38 \\
\hline
\end{tabular}

\section{CONCLUSIONES}

De acuerdo a los resultados obtenidos, la codificación LDPC irregular tiene un muy buen desempeño en un canal de línea eléctrica de baja tensión, obteniéndose una ganancia de más de $30 \mathrm{~dB}$ en comparación con el código de línea para una velocidad de transmisión de 3 a $30 \mathrm{Mbps}$ para obtener un BER de $10^{-6}$.

La característica impulsiva del ruido en la línea eléctrica de baja tensión depende directamente del número de fuentes presente en la línea eléctrica.

Un futuro trabajo de investigación es la combinación de código LDPC irregular con entrelazador de bit, porque el entrelazador de bit muestra un buen desempeño en errores de ráfaga (ruido impulsivo).

\section{AGRADECIMENTOS}

Los autores de este trabajo agradecen a la Universidad del Bío-Bío, Concepción-Chile, por apoyar y financiar el proyecto de investigación código 052210 3/R.

\section{REFERENCIAS}

[1] D.G. Fink. "Standard Handbook for Electrical Engineers", Chapter 13-24, McGraw-Hill. 2000.

[2] K. Dostert. "Powerline Communications". PrenticeHall. 2001.

[3] R.C. Cheek. "Electrical Transmission and Distribution”. Chapter 12, Westinghouse Electric Corp. 1999.

[4] N. Biju. "Telecommunications for Deregulated Power Industry". Master Thesis, Virginia University. 2002.

[5] G. Platt. "Domestic Powerline Carrier Communications". Thesis Bachelor of Engineering in Electrical Engineering, October 1999.

[6] L. Selander. "Powerline Communications-Channel Properties and Communications Strategies". PhD thesis, Lund University. 1999.

[7] K.H. Zuberi. "Powerline Carrier Communications Systems". MS Thesis, Departament of Microelectronics and Information Technology, Royal Institute of Technology, KTH. 2003.

[8] K. Dostert. "EMC Aspects of High Speed Powerline Communications". Proceedings of $15^{\text {th }}$ International Wroclaw Symposium and Exhibition on Electromagnetic Compatibility, Wroclaw, Poland. June 27-30. 2000.

[9] K. Dostert. "Frequency Hopping Spreed Spectrum Modulation for Digital Communications over Electrical Powerlines", IEEE Journal on Selectred areas in Communications. Vol. $9 \mathrm{~N}^{\mathrm{o}} 3$, pp. 700-710. May 1990.

[10] O.G. Hooijen. "A Channel Model for Residential Power Circuit Used as a Digital Communication Medium". IEEE Transaction on Electromagnetic Compatibility. Vol. $40 \mathrm{~N}^{\circ}$ 4, pp. 331-336. November 1998.

[11] M. Zimmermann and K. Dostert. "A Multipath Model for Powerline Channel”. IEEE Transactions on Communications. Vol. $50 \mathrm{~N}^{\mathrm{o}} 4$, pp. 553-559. April 2002. 
[12] R.G. Gallager. "Low Density Parity Check Codes". Research monograph series. No 21. MIT Press, Cambridge Mas. 1963.

[13] M.C. Davey and D.J. Mackay. "Low Density Parity Check Codes Over GP(q)". Proceedings of 1998 IEEE Information Theory Worshop. June 1998.

[14] M.G. Luby, M.A. Shokrollahi. "Improved Low Density Parity Check Codes Using Irregular Graphs and Belief Propagation". Proceedings of the 1998 IEEE International Symposium on Information Theory, p. 117. Boston, U.S.A.

[15] D.A. Spielman. "Linear Time Encodable and Decodable Error Correcting Codes". IEEE Transactions on Information Theory. Vol. IT-42 No 6, pp. 1723-1731. 1996.

[16] B.J. Frey and F.R. Kschischang. "Probability Propagation and Iterative Decoding". Proceedings of the 34th Allerton Conference on Communications, Control and Computing. 1996.

[17] N. Wiberg, "Codes and Decoding on General Graphs". PhD, Dept. Elec. Eng. University Linkoping, Sweeden. April 1996.

[18] J. M. Selga. "PLC, Internet por la red eléctrica". Julio 2002. URLs: www.alcion.es

[19] M. Zimmerman and K. Dostert. "Analysis and Modeling of Impulsive in Broad Band Powerline Communications". IEEE Transactions on Electromagnetic Compatibility. Vol. $44 \mathrm{~N}^{\mathrm{o}} 1$, pp. 249-258. February 2002.

[20] B.D. Frichman. "A Binary Channel Characterization Using Partitioned Markov Chains". IEEE Transactions Information Theory. Vol. IT-13. April 1967.

[21] S.Y. Jung. "A Channel Model for Powerline Communications in Home Network". Proceedings on the $15^{\text {th }}$ CISL Winter Workshop, Kushu, Japan. February 2002.

[22] D. Midleton. "Canonical Non-Gaussian Models: Their Implications for Measurement and for Prediction Receiver Performance". IEEE Transactions of Communications. Vol. COM-21, №3, pp. 20-220. August 1979.

[23] K. Yamauchi and N. Takahashi. "Parameter Measurements of Class A Interferente on Powerline". Transactions IICE. Vol. $72 \mathrm{~N}^{\mathrm{o}}$ 1, pp. 7-9. January 1989.

[24] W. Fernández. "Combinación de códigos LDPC irregulares con canal Mimo para 4G”. Tesis para optar al grado de Magíster. Departamento de Ingeniería Eléctrica. Universidad de Santiago de Chile. Santiago, Chile. 2003.

[25] R.M. Tanner. "A Recursive Approach to Low Complexity Codes". IEEE Transactions Information Theory. Vol. IT-27 No 5, pp. 533-547. September 1981. 